\title{
Moscas-das-frutas (Diptera: Tephritidae) e seus hospedeiros no município de Quixeré, estado do Ceará, Brasil
}

\section{Fruit flies (Diptera: Tephritidae) and their hosts in the municipality of Quixeré, state of Ceará, Brazil}

\author{
Marcia Mayara de Sousa ${ }^{1}$; Daniele Campos Martins ${ }^{2}$; Elania Clementino Fernandes ${ }^{3}$; Antonia Débora Camila de Lima \\ Ferreira $^{4}$; Elton Lucio Araujo ${ }^{5 *}$
}

\begin{abstract}
Resumo: O estado do Ceará é um dos principais produtores e exportadores de frutas tropicais do Brasil. No entanto, os produtores têm alguns problemas relacionados com as moscas-das-frutas (Diptera: Tephritidae), pois estes tefritídeos causam danos aos frutos e a simples presença de algumas espécies dificulta a exportação de frutas in natura. No estado do Ceará são escassas as informações sobre as moscas-das-frutas e seus hospedeiros nas regiões produtoras de frutas, como por exemplo, na região do Baixo Jaguaribe. Esta região está localizada no semiárido brasileiro e é composta por dez municípios, entre eles o município de Quixeré. Portanto, o objetivo deste trabalho foi conhecer as espécies de moscas-das-frutas, seus hospedeiros e respectivos índices de infestação, em diferentes locais do município de Quixeré. Para isto, foram realizadas coletas aleatórias de frutos em diferentes frutíferas (nativas e exóticas), na zona rural e urbana de Quixeré. Os frutos coletados eram levados para o laboratório, onde eram contados, pesados e acondicionados em bandejas plásticas sobre uma camada de vermiculita. Após sete dias, a vermiculita era peneirada e os pupuários obtidos eram acondicionados em recipientes plásticos até a emergência dos adultos. Frutos de 22 espécies foram amostrados e apenas cinco estavam infestadas por moscas-das-frutas. As espécies obtidas foram Ceratitis capitata (Wiedemann), Anastrepha zenildae Zucchi, Anastrepha sororcula Zucchi e Anastrepha obliqua (Macquart). A goiaba Psidium guajava L. foi o fruto que apresentou os maiores índices de infestação.
\end{abstract}

Palavras-chave: Insecta; Frugívoros; Biodiversidade; Semiárido; Bioma Caatinga.

\begin{abstract}
The state of Ceará is one of the main producers and exporters of tropical fruits in Brazil. However, the farmers have some problems related with the fruit flies (Diptera: Tephritidae), because these tefritids cause damages to the fruits and the simple presence of some species makes difficult the export of fruits in natura. In the state of Ceará, information about fruit flies and their hosts in fruit producing regions are scarce, such as in the region of Baixo Jaguaribe. This region is located in the Brazilian semiarid and is composed of ten municipalities, among them the municipality of Quixeré. Therefore, the objective of this study was to know the species of fruit flies, their hosts and respective infestation index, in different places of the municipality of Quixeré. For this, fruits were randomly collected in different fruit trees (native and exotic), in the rural and urban area of Quixeré. The collected fruits were transported to the laboratory, where they were counted, weighed and stored in plastic trays on a layer of vermiculite. After seven days, the vermiculite was sieved and the pupae obtained were stored in plastic containers until the emergence of adults. Fruits of 22 species were sampled and only five were infested by fruit flies. The species obtained were Ceratitis capitata (Wiedemann), Anastrepha zenildae Zucchi, Anastrepha sororcula Zucchi and Anastrepha obliqua (Macquart). Guava Psidium guajava L. was the fruit that presented the highest rates of infestation.
\end{abstract}

Key words: Insecta; Frugivorous; Biodiversity; Semiarid; Caatinga Biome.

\footnotetext{
*Autor para correspondência

Recebido para publicação em 07/04/2017; aprovado em 20/06/2017

${ }^{1}$ Mestre em Entomologia pela Universidade Federal Rural de Pernambuco (UFRPE), Recife-PE; E-mail: marciamayarasos@gmail.com

${ }^{2}$ Graduação em Agronomia pela Universidade Federal Rural do Semi-Árido (UFERSA), Mossoró-RN, E-mail: daniele_martins@ @otmail.com

${ }^{3}$ Mestre em Fitotecnia/Proteção de Plantas pela Universidade Federal Rural do Semi-Árido (UFERSA), Mossoró-RN, E-mail: elania.fernandes@ ufersa.edu.br

${ }^{4}$ Mestre em Agronomia/Fitotecnia pela Universidade Federal do Ceará (UFC), Fortaleza-CE, E-mail: camilaferreira1@ hotmail.com

${ }^{5}$ Doutor, Professor Associado da Universidade Federal Rural do Semi-Árido (UFERSA), Mossoró-RN, E-mail: elton@ufersa.edu.br
} 


\section{INTRODUÇÃO}

O Brasil é um dos maiores produtores de frutas tropicais do mundo, sendo o estado do Ceará, um dos que mais se destaca na exportação de frutas in natura (ABF, 2017). Uma das principais regiões produtoras de frutas do Ceará é a região denominada de Baixo Jaguaribe, que está inserida no bioma Caatinga (semiárido brasileiro) e é constituída por dez municípios, dentre eles o município de Quixeré (IBGE, 2016).

Apesar da relevância da fruticultura para região do Baixo Jaguaribe, os produtores têm problemas para produção e comercialização de algumas frutas, devido os danos causados pelas moscas-das-frutas (Diptera: Tephritidae). As larvas destes tefritídeos se alimentam da polpa dos frutos, tornando-os impróprios para o consumo e industrialização (WHITE; ELSON-HARRIS, 1992). Além disso, algumas espécies de moscas-das-frutas são importantes do ponto de vista quarentenário, devido a isso, determinados países mais exigentes evitam importar frutas de regiões do mundo onde estas espécies de importância quarentenária ocorrem, para evitar a introdução de pragas em seus territórios (ZUCCHI, 2015). No Brasil, as moscas-das-frutas de importância econômica e quarentenária pertencem aos gêneros Anastrepha e Ceratitis, este último representado apenas por uma espécie, Ceratitis capitata (Wiedemann) (ZUCCHI, 2017).

Para se estabelecer um programa de manejo integrado das moscas-das-frutas de maneira adequada, em uma determinada região, é fundamental conhecer as espécies de moscas-das-frutas e seus hospedeiros presentes na região de interesse (ARAUJO et al., 2005). Contudo, no estado do Ceará, os levantamentos de moscas-das-frutas em seus hospedeiros são escassos, e a maioria deles foi realizado de maneira pontual. Souza et al. (2008) realizaram um levantamento no campus central da Universidade Federal do Ceará (UFC), situado na cidade de Fortaleza, e observaram nove frutíferas como hospedeiras de três espécies de moscasdas-frutas. Posteriormente, também no campus central da UFC, Moura e Moura (2011) relataram as espécies Anastrepha zenildae Zucchi, Anastrepha sororcula Zucchi e C. capitata infestando frutos de goiaba.

Com relação à região do Baixo Jaguaribe, as informações existentes sobre moscas-das-frutas foram obtidas através de levantamentos com armadilhas. Dessa maneira, Araujo et al. (2009) relataram a ocorrência de sete espécies de moscas-das-frutas na região do Baixo Jaguaribe: A. zenildae, A. sororcula, Anastrepha obliqua (Macquart), Anastrepha daciformis Bezzi, Anastrepha pickeli Lima, Anastrepha consobrina (Loew) e C. capitata, mas no município de Quixeré apenas A. zenildae, A. sororcula e A. obliqua foram registradas. No entanto, nos levantamentos realizados com uso de armadilhas não é possível conhecer os diferentes tipos de frutíferas que são hospedeiras de moscas-das-frutas, consequentemente não se pode verificar quais frutíferas apresentam as maiores e menores infestações.

Portanto, devido os poucos levantamentos de espécies de moscas-das-frutas e à falta de informações sobre os hospedeiros e índices de infestação destes tefritídeos nas regiões produtoras de frutas do estado do Ceará, o objetivo deste trabalho foi conhecer as espécies de moscas-das-frutas, seus hospedeiros e índices de infestação, no município de Quixeré, situado no semiárido brasileiro.

\section{MATERIAL E MÉTODOS}

As coletas de frutos foram realizadas em diversas localidades pertencentes à zona urbana e à zona rural do município de Quixeré (0504'26” S e 3759'20" O, 30 metros de altitude), Ceará, Brasil. O levantamento foi realizado durante o período de agosto de 2011 a julho de 2012. O município de Quixeré está localizado em uma região do semiárido brasileiro, conhecida como Baixo Jaguaribe, onde predomina o clima semiárido quente com temperatura média anual de $27,7^{\circ} \mathrm{C}$, média pluviométrica anual em torno de 800 mm e a vegetação predominante é do tipo Caatinga (IBGE, 2016; CLIMATE-DATA, 2017).

Os frutos foram coletados de maneira aleatória em diversas localidades. Foram amostrados frutos pertencentes a 22 espécies frutíferas: acerola Malpighia emarginata D.C. (13 amostras/10,9 Kg), goiaba Psidium guajava L. (31 amostras/36,1 Kg), romã Punica granatum L. (3 amostras/0,6 $\mathrm{Kg}$ ), manga Mangifera indica L. (12 amostras/26,5 Kg), seriguela Spondias purpurea L. (7 amostras/3,1 Kg), sapoti Manilkara zapota (L.) (2 amostras/1,5 Kg), condessa Annona reticulata L. (1 amostra/0,6 Kg), mutamba Guazuma ulmifolia Lam. (3 amostras/1,1 Kg), cajarana Spondias sp. (3 amostras $/ 5,9 \mathrm{Kg}$ ), cajá Spondias mombin L. (2 amostras/1,3 $\mathrm{Kg}$ ), graviola Annona muricata L. (3 amostras/1,3 Kg), castanhola Terminalia catappa L. (2 amostras/1,0 $\mathrm{Kg})$, mamão Carica papaya L. (3 amostras $/ 8,7 \mathrm{Kg}$ ), tangerina Citrus reticulata Blanco (4 amostras $/ 0,9 \mathrm{Kg}$ ), lima Citrus limettioides Tan. (3 amostras/1,5 Kg), laranja Citrus sinensis (L.) (5 amostras $/ 5,5 \mathrm{Kg}$ ), jambo Syzygium jambos (L.) (2 amostras/1,5 Kg), carnaúba Corpenicia prunifera (Mill.) (2 amostras/0,5 Kg), noni Morinda citrifolia L. (1 amostra/0,3 $\mathrm{Kg}$ ), abacate Persea americana Mill. (1 amostra/0,3 Kg), carambola Averrhoa carambola L. (1 amostra/0,6 Kg) e juá Ziziphus joazeiro Mart. (3 amostras/3,3 Kg).

$\mathrm{Na}$ ocasião das coletas, as amostras de cada tipo de fruto eram acondicionadas em sacos de papel devidamente identificados e transportados para o Laboratório de Entomologia Aplicada/Centro de Ciências Agrárias da Universidade Federal Rural do Semi-Árido (UFERSA), em Mossoró, Rio Grande do Norte.

No laboratório, os frutos eram pesados, contados e colocados em bandejas plásticas sobre uma camada de vermiculita fina, onde permaneciam por um período de sete a dez dias. Após este período, a vermiculita era peneirada para obtenção dos pupários, que eram contabilizados, acondicionados em placas de Petri e cobertas com filme plástico. Os pupários permaneciam nas placas até a emergência dos adultos. As moscas-das-frutas emergidas eram acondicionadas em recipientes plásticos, contendo álcool a $70 \%$, onde permaneciam até a devida identificação específica.

$\mathrm{O}$ índice de infestação dos frutos foi calculado através da seguinte fórmula: Índice de Infestação $=\left(\mathrm{N}^{\mathrm{o}}\right.$ de pupários obtidos/Kg de frutos).

O reconhecimento das espécies de frutíferas foi realizado com base na literatura e nas informações disponíveis no herbário Dárdano de Andrade Lima, situado na UFERSA. As moscas-das-frutas foram identificadas com base no padrão alar, coloração do corpo e características morfométricas do ápice do acúleo das fêmeas (ZUCCHI, 2000; ARAUJO; ZUCCHI, 2006). 


\section{RESULTADOS E DISCUSSÃO}

Neste estudo foram coletados um total de 6.423 frutos, correspondendo a 117,3 Kg. Entre as 22 espécies de frutíferas amostradas, cinco (acerola, goiaba, laranja, juá e seriguela) estavam infestadas por pelo menos uma espécie de moscasdas-frutas (Tabela 1). Dos frutos infestados foram obtidos 569 pupários, dos quais emergiram 469 adultos de moscas-das-

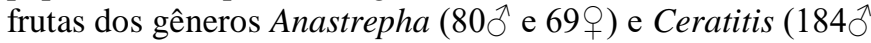
e 136ㅇ). As moscas-das-frutas obtidas pertencem às espécies: A. sororcula, A. zenildae, A. obliqua e C. capitata. Dentre as espécies constatadas, A. obliqua e $C$. capitata são consideradas de importância quarentenária para vários países (WHITE; ELSON-HARRIS, 1992; MALAVASI et al, 2000; ZUCCHI, 2015). Dessa maneira, para se exportar frutas in natura que são hospedeiras dessas duas espécies de tefritídeos, certamente serão exigidas medidas fitossanitárias mais rigídas (por exemplo, o tratamento hidrotérmico em manga), por parte de alguns países importadores.

As espécies de moscas-das-frutas obtidas são comuns no semiárido brasileiro e já haviam sido registradas na região do Baixo Jaguaribe, Ceará (ARAUJO et al., 2009). No entanto, estes são os primeiros relatos de $C$. capitata no município de Quixeré, como também, os primeiros registros de frutos hospedeiros de moscas-das-frutas neste município. A espécie C. capitata foi a mais comum neste levantamento $(68,2 \%)$ e foi observada em quatro dos cinco tipos de frutos infestados pelas moscas-das-frutas (Tabela 1). De acordo com Szyniszewska e Tatem (2014), C. capitata é altamente polífaga e cosmopolita. Por outro lado, as espécies de Anastrepha foram encontradas em menor quantidade $(31,8 \%)$ e infestaram apenas dois dos cinco tipos de frutos registrados como hospedeiros de moscas-das-frutas. $\mathrm{O}$ fato de $C$. capitata ter sido observada em um maior número de hospedeiros chama a atenção, pois esse tefritídeo é exótico e só foi detectado na região Nordeste do Brasil em 1991 (ZUCCHI, 2015).
A espécie $C$. capitata foi obtida em maior número em frutos de goiaba (Tabela 1). Estes resultados reforçam as informações de que $C$. capitata tem status de praga de goiabeira nessa região do semiárido (ARAUJO et al., 2013). Este tefritídeo também foi comum em frutos de acerola, inclusive foi a única espécie associada à acerola. Em outras regiões do Brasil, frutos de acerola são considerados hospedeiros de C. capitata (ZUCCHI, 2017). Além disso, a ocorrência deste tefritídeo em acerola pode ter relação com o fato do maior número de coleta desse fruto ter sido realizado na zona urbana de Quixeré. Segundo Alvarenga et al. (2010), C. capitata predomina em áreas urbanas. Poucos exemplares de C. capitata foram obtidos em frutos de laranja e seriguela, no entanto, $C$. capitata foi à única espécie associada a estes dois hospedeiros. Na região Sudeste do Brasil, principalmente no estado de São Paulo, onde se encontram as maiores áreas cultivadas com citros no país, $C$. capitata é considerada uma praga chave para a citricultura (RAGA, 2005). As informações deste trabalho mostram que $C$. capitata é uma potencial praga de citros em ambiente semiárido. De uma maneira geral, a maioria dos frutos infestados por C. capitata são exóticos, revelando a preferência deste tefritídeo por frutíferas exóticas como relatado por Araujo et al. (2005).

As espécies de Anastrepha relatadas neste estudo foram obtidas basicamente em frutos de goiaba, apenas A. zenildae foi encontrada em frutos de goiaba e juá (Tabela 1). A associação de $A$. sororcula e A. zenildae com frutos de goiaba não surpreendeu, pois estas duas espécies são comuns em goiaba, em regiões do semiárido brasileiro (ALVARENGA et al., 2009). Apenas um exemplar de A. obliqua foi constatado nos frutos de goiaba amostrados, confirmando que A. obliqua não é comum em goiaba nessa região do semiárido (ARAUJO et al., 2005). A incidência de A. zenildae em frutos de juá é comum no semiárido, onde praticamente é a única espécie que explora frutos de juá como recurso alimentar (ARAUJO et al., 2005; QUERINO et al., 2014).

Tabela 1. Número de exemplares de moscas-das-frutas (Anastrepha e Ceratitis) obtidos em frutos coletados no município de Quixeré, Ceará, no período de agosto de 2011 a julho de 2012.

\begin{tabular}{lcccc}
\multicolumn{1}{c}{ Hospedeiros } & \multicolumn{3}{c}{ Espécies de moscas-das-frutas } \\
\cline { 2 - 5 } & C. capitata & A. sororcula & A. zenildae & A. obliqua \\
\hline Acerola Malpighia emarginata D.C. (Malpighiaceae) (E) & 32 & - & - & - \\
Goiaba Psidium guajava L. (Myrtaceae) (N) & 96 & 55 & 6 & - \\
Laranja Citrus sinensis (L.) (Rutaceae) (E) & 7 & - & - & - \\
Juá Ziziphus joazeiro Mart. (Rhamnaceae) (N) & - & - & - \\
Seriguela Spondias purpurea L. (Anacardiaceae) (E) & 1 & - & - \\
\hline
\end{tabular}

(N) Nativo; (E) Exótico

Os maiores índices de infestação de moscas-das-frutas foram observados em frutos de goiaba (12,2 pupários/Kg de frutos) e acerola (9,8 pupários/Kg de frutos) (Figura 1). As maiores infestações em goiaba e acerola demonstram que estes frutos são hospedeiros preferenciais de moscas-dasfrutas em Quixeré. Araujo et al. (2005) relataram que goiaba e acerola estão entre os frutos mais infestados por Anastrepha spp. e $C$. capitata, respectivamente, em ambientes semiárido. Araujo et al. (2011) constataram índices de infestação de $C$. capitata de 28 (pupários $/ \mathrm{Kg}$ de frutos) em pomares comerciais de acerola comum, em uma região semiárida do Rio Grande do Norte. Em outras regiões do país, goiaba e acerola também são considerados bons hospedeiros de moscas-das-frutas. Garcia e Norrbom (2011) relataram índices de infestação de 116,64 (pupários/Kg de fruto) em goiaba, na região Sul do Brasil e Raga et al. (2011) verificaram um índice de infestação de 21,78 (pupários/Kg de fruto) em acerola, na região Sudeste do país.

As menores infestações foram constatadas em frutos de juá (2,3 pupários/Kg de fruto), laranja (2,2 pupários/Kg de fruto) e seriguela (0,3 pupários/Kg de fruto) (Figura 1). As baixas infestações de mosca-das-frutas em frutos de juá e seriguela chamou a atenção, pois geralmente estes frutos são bem infestados por A. zenildae e C. capitata, respectivamente, nessa região do semiárido (ARAUJO et al., 2005). Estes autores relataram índices de infestação de 67,7 (pupários/Kg de fruto) de A. zenildae em frutos de juá e de 34,5 (pupários/Kg de fruto) de C. capitata em frutos de seriguela, 
em uma região semiárida do Rio grande do Norte, próxima de onde foi realizado o presente estudo. O baixo índice de infestação de moscas-das-frutas em frutos de laranja possivelmente foi devido à presença de laranjeiras não ser comum na região, no entanto, com o passar do tempo e o aumento da área plantada é provável que as populações de $C$. capitata passem a infestar frutos de laranja com maior intensidade.

Figura 1. Índice de infestação de moscas-das-frutas (Anastrepha e Ceratitis) em frutos coletados no município de Quixeré, Ceará, no período de agosto de 2011 a julho de 2012.

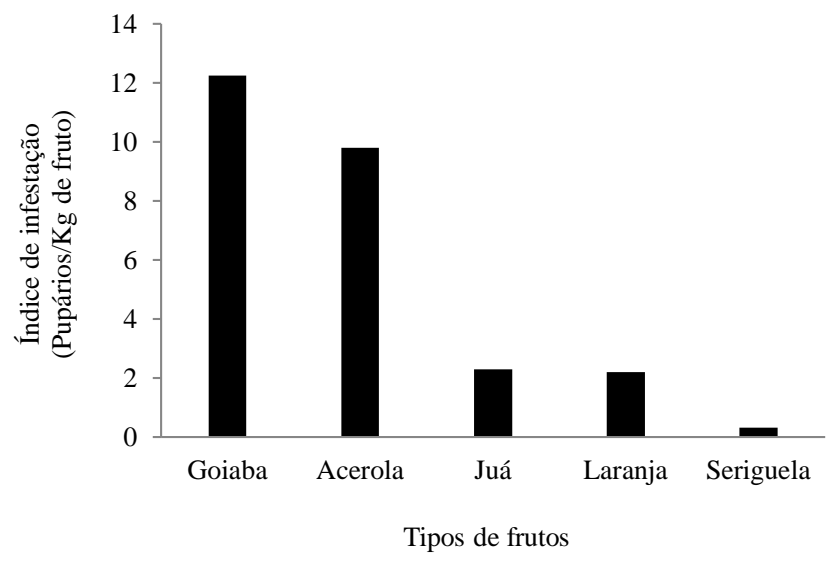

De uma maneira geral, os resultados obtidos neste trabalho demonstram que entre as quatro espécies de moscasdas-frutas obtidas, $C$. capitata foi a mais comum e a que infestou a maior diversidade de hospedeiros, sendo a maioria dos frutos hospedeiros de origem exótica. Apesar da maior quantidade de frutos infestados serem exóticos, a goiaba (nativa) foi à fruta que hospedou o maior número de espécies de moscas-das-frutas e a que apresentou o maior índice de infestação, demonstrando ser um hospedeiro preferencial para as espécies de moscas-das-frutas que ocorrem no município de Quixeré. Portanto, estas informações sugerem que para o manejo integrado de moscas-das-frutas em pomares de frutíferas no município de Quixeré, semiárido brasileiro, as maiores atenções devem se concentrar na $C$. capitata, pois é uma espécie comum nessa região, principalmente em associação com frutos exóticos, que são hospedeiros de moscas-das-frutas.

\section{CONCLUSÕES}

As espécies de moscas-das-frutas detectadas no município de Quixeré, Ceará foram: Anastrepha sororcula, A. zenildae, A. obliqua e Ceratitis capitata.

Os frutos registrados como hospedeiros de moscas-dasfrutas (Anastrepha spp e C. capitata) foram: goiaba Psidium guajava, acerola Malpighia emarginata, juá Ziziphus joazeiro, laranja Citrus sinensis e seriguela Spondias purpurea.

Os índices de infestação registrados nos hospedeiros conhecidos foram: goiaba (12,2 pupários/Kg de frutos), acerola (9,8 pupários/Kg de frutos), juá (2,3 pupários/Kg de fruto), laranja (2,2 pupários/Kg de fruto) e seriguela $(0,3$ pupários/Kg de fruto).

\section{REFERÊNCIAS}

ABF, Anuário Brasileiro da Fruticultura. Beling, R. R. (Ed.), Editora Gazeta Santa Cruz, 2017. 88p.

ALVARENGA, C. D.; MATRANGOLO, C. A. R.; LOPES, G. N.; SILVA, M. A.; LOPES, E. N.; ALVES, D. A.; NASCIMENTO, A. S.; ZUCCHI, R. A. Moscas-das-frutas (Diptera: Tephritidae) e seus parasitóides em plantas hospedeiras de três municípios do norte do estado de Minas Gerais. Arquivos do Instituto Biológico, v.76, n.2, p.195-204, 2009.

ALVARENGA, C. D.; ALVES, D. A.; SILVA, M. A.; LOPES, E. N.; LOPES, G. N. Moscas-das-frutas (Diptera: Tephritidae) em pomares da área urbana no norte de Minas Gerais. Revista Caatinga, v.23, n.2, p.25-31, 2010.

ARAUJO, E. L.; MEDEIROS, M. K. M.; SILVA, V. E.; ZUCCHI, R. A. Moscas-das-frutas (Diptera: Tephritidae) no semi-árido do Rio Grande do Norte: plantas hospedeiras e índices de infestação. Neotropical Entomology, v.34, n.6, p.889-894, 2005.

ARAUJO, E. L.; ZUCCHI, R. A. Medidas do acúleo na caracterização de cinco espécies de Anastrepha do grupo fraterculus (Diptera: Tephritidae). Neotropical Entomology, v.35, n.3, p.329-337, 2006.

ARAUJO, E. L.; CUNHA, A. A.; SILVA, R. K. B.; NUNES, A. M. M.; GUIMARÃES, J. A. Espécies de moscas-dasfrutas (Diptera: Tephritidae) na região do Baixo Jaguaribe, estado do Ceará. Arquivos do Instituto Biológico, v.76, n.4, p.577-581, 2009.

ARAUJO, E. L.; LOPES, P. A. R.; SILVA, J. G.; BITTENCOURT, M. A. L.; RONCHI-TELES, B. Índices de captura e infestação da mosca do mediterrâneo em acerola comum e clonada. Revista Verde, v.6, n.4, p.58-64, 2011.

ARAUJO, E. L.; RIBEIRO, J. C.; CHAGAS, M. C. M.; DUTRA, V. S.; SILVA, J. G. Moscas-das-frutas (Diptera: Tephritidae) em um pomar de goiabeira, no semiárido brasileiro. Revista Brasileira de Fruticultura, v.35, n.2, p.471476, 2013.

CLIMATE-DATA. Disponível em: <http://pt.climatedata.org/location/42566/ >. Acesso: Outubro. 2017.

GARCIA, F. R. M.; NORRBOM, A. Tephritoid flies (Diptera, Tephritoidea) and their plant hosts from the State of Santa Catarina in Southern Brazil. Florida Entomologist, v.94, n.2, p.151-157, 2011.

IBGE, Instituto Brasileiro de Geografia Estatística. Pesquisa $2016 . \quad$ Disponível em: <http://www.cidades.ibge.gov.br/brasil/ce/quixere/panorama> Acesso: Outubro. 2017.

MALAVASI, A.; ZUCCHI, R. A.; SUGAYAMA, R. Biogeografia. In: MALAVASI, A.; ZUCCHI, R. A. (Eds). Moscas-das-frutas de importância econômica no Brasil. 
Conhecimento básico e aplicado. Ribeirão Preto: Holos Editora, 2000. cap. 10, p.93-98.

MOURA, A. P; MOURA, D. C. M. Levantamento e flutuação populacional de parasitoides de moscas-das-frutas (Diptera: Tephritidae) de ocorrência em goiabeira (Psidium guajava L.) em Fortaleza, Ceará. Arquivos do Instituto Biológico, v.78, n.2, p.225-231, 2011.

QUERINO, R. B.; MAIA, J. B.; LOPES, G. N.; ALVARENGA, C. D.; ZUCCHI, R. A. Fruit fly (Diptera: Tephritidae) community in guava orchards and adjacent fragments of native vegetation in Brazil. Florida Entomologist, v.97, n.2, p.778-786, 2014.

RAGA, A. Incidência, monitoramento e controle de moscasdas-frutas na citricultura paulista. Laranja, v.26, n.2, p.307322, 2005.

RAGA, A.; SOUZA-FILHO, M. F.; MACHADO, R. A.; SATO, M. E.; SILOTO, R. C. Host ranges and infestation indices of fruit flies (Tephritidae) and lance flies (Lonchaeidae) in São Paulo State, Brazil. Florida Entomologist, v.94, n.4, p.787-794, 2011.

SOUZA, A. J. B.; LIMA, M. G. A.; GUIMARÃES, J. A.; FIGUEIREDO, A. E. Q. Moscas-das-frutas (Diptera:
Tephritidae) associadas às plantas hospedeiras do pomar do campus do PICI da Universidade Federal do Ceará. Arquivos do Instituto Biológico, v.75, n.1, p.21-27, 2008.

SZYNISZEWSKA, A. M.; TATEM, A. J. Global assessment of seasonal potential distribution of mediterranean fruit fly, Ceratitis capitata (Diptera: Tephritidae). PlosOne, v.9, n.11, p.e111582, 2014.

WHITE, I. M.; ELSON-HARRIS, M. M. Fruit flies of economic significance: their identification and bionomics. Wallingford: CAB International, 1992. 601p.

ZUCCHI, R. A. Taxonomia. In: MALAVASI, A.; ZUCCHI, R. A. (Eds). Moscas-das-frutas de importância econômica no Brasil. Conhecimento básico e aplicado. Ribeirão Preto: Holos Editora, 2000. cap. 1, p.13-24.

ZUCCHI, R. A. Mosca-do-mediterrâneo, Ceratitis capitata (Wiedemann). In: VILELA, E. F.; ZUCCHI, R. A. (Eds). Pragas introduzidas no Brasil: insetos e ácaros. Piracicaba: FEALQ, 2015. 908p..

ZUCCHI, R. A. Fruit flies in Brazil: Anastrepha species and their hosts plants. Piracicaba: ESALQ. 2017. Disponível em: <http://www.lea.esalq.usp.br/anastrepha/> Acesso em: Novembro. 2017. 